\title{
DAB-Restorative Simplicity
}

\section{Ron Carlson}

Carlson Bridge Technologies, Inc. Private Enterprise, Hawaiia, USA

*Corresponding author: Ron Carlson, Carlson Bridge Technologies, Inc. Private Enterprise, Hawaiia, USA, Tel: 01-808-735-0282; E-mail: dscarlson@hawaiiantel.net

Received date: September 29, 2017, Accepted date: October 04, 2017, Published date: October 09, 2017

Copyright: (c) 2017 Carlson R. This is an open-access article distributed under the terms of the Creative Commons Attribution License, which permits unrestricted use, distribution, and reproduction in any medium, provided the original author and source are credited.

\begin{abstract}
An all-composite Direct Adherence Bridge (Direct Adhesion Prosthesis) is a fixed dental replacement for one or multiple missing teeth that need replacing, relies on its adherence and strength, with superior composites, and its adherence with attachment components_bonding agents. It is the newest of the five currently available methods for replacing missing teeth. The four most commonly in use are the: 1) "flipper," a removable prosthesis; 2) the orthodox fixed bridge with metal requiring tooth preparation; 3) the resin retained bridge with specific metal and design requirements; and, 4) the dental implant-all requiring multiple appointments and tissue alteration-the DAB does not. All previous methods are of an "indirect nature" having laboratory phases; the DAB is a "direct intervention." A direct adherence prefabricated dental bridge has no metal framework and requires none. It is simply a prefabricated false tooth made of composite that may be altered to fit the space between two (or alongside one) natural teeth that is bonded in place once adjusted. The Direct Adherence Bridge (glue-in bridge) is only adhered, in most cases, to external structures of natural teeth or silicate porcelain veneer crowns over altered teeth; and, will not damage the surrounding teeth due to preparation (cutting hard structures) or placement and is well accepted and liked and preferred by patients. The average life span of a-glue in bridge is that of any other fixed bridge. The adherence material that holds the winged pontic to the natural structures is the same composite, which composes the pontic. The composite to tooth bond strength is approximately $4,800 \mathrm{lbs} / \mathrm{in}^{2}$, which far exceeds all resin, or conventional adhesion cements which range from 1,500 psi to 2,240 psi. The compressive strength of the composite is about $62,000 \mathrm{psi}$ and its tensile strength about 22,000 psi.
\end{abstract}

Keywords: Direct adherence bridge; Conventional bridges; Implant; Enamel

\section{Introduction}

The DAB is also devoid of gross fiber inclusion since macro-fibers such as Ribbond, Kevlar, or Nylon mesh do not do what they purported are said to do-make the composite matrix stronger [1-3]. DAB is simply a prefabricated false tooth made of dental composite paste of extraordinary quality that may be altered to fit the space between two (or alongside one) natural teeth that is bonded in place once adjusted. The Direct Adherence Bridge (glue-in bridge, DAB) is only adhered, in most cases, to external structures of natural teeth or silicate porcelain veneer crowns over altered teeth; and, will not damage the surrounding teeth due to preparation (cutting hard structures) or placement and is well accepted and preferred by patients. The average life span of a-glue in bridge is that of any other fixed bridge. Insurance actuarial average is five years.

In conventional bridges the failure is likely to be complete fracture of the abutment tooth with difficult-to-manage results, possibly requiring extraction of its abutment, while the glue-in methodology can be easily repaired and reattached in situ. A concept similar to the "glue-in fixed bridge" (DAB) is the resin-retained bridge, but the latter has many drawbacks. Unlike the DAB, glue-in dental bridge, the resinretained bridge, such as the Maryland bridge or the Rochette bridge, the resin-retained bridge requires a very specific set of design principles, cutting of enamel and or dentine and multiple appointments since the metal framework is constructed in a laboratory utilizing an indirect methodology. Porcelain fused to metal Maryland and Rochette bridges contain beryllium metal that is a known carcinogen. This is why the vast majority of dentists use for their Maryland bridges now made of porcelain such as e-max or zirconia. These are becoming more common but require more thickness to be strong enough. Zirconia Maryland bridges can be very good but one must worry about how well you are bonding to the tooth structure with zirconia.

Direct Adhesion Bridges require one appointment only for preparation of the teeth (without cutting), or porcelain crowns over them, and of the materials for complete installation and finishing [4,5]. The direct insertion of the direct adhesion fixed bridge (the glue-in "winged pontic") is accomplished after adjustment of the false tooth to fit the space and then attachment components added to develop the wings which are finalized and augmented, bonding them to the natural teeth or old porcelain veneer crowns as one builds the direct bridgethus the term direct glue-in "winged pontic" fixed bridge as distinguished from the indirect methodology of all other methods employed in tooth replacement. We present a comparison chart of the various methods presented in the table below feeling that once reviewed it will assist the practitioner with valuable and pertinent information and distinctions.

\section{Installation Process}

The direct adherence bridge methodology of bridging takes place in the following sequences:

- Teeth are scrupulously cleaned and then etched with about a $38 \%$ phosphoric-nitric acid (Ultra Dent) solution for about 1 minute. 
Page 2 of 4

- The "winged pontic" had been previously adjusted so that it easily slides into the space in a reproducibly way.

- The winged pontic is treated the same as the teeth with acid.

- Both the teeth and winged pontic are cleaned with water, dried and then silanated (Carlson Bridge WP Bond Enhancer) and a clear polymer (Carlson Bridge WP Clear Resin Adhesive) applied to a glossy sheen.

- Soft dental composite (Carlson Bridge WP Composite) is applied to the proximals of the winged pontic and to one or two proximals of the attachment teeth.

- The prefabricated winged pontic direct adhesion bridge is installed in proper alignment and after confirming occlusion and line of draw, cured with the polymerizing blue light-visible light curing (TPC LED55, 440 nM-490 nM [TPC Advanced Technology]).

- Additional composite is added as needed (Carlson Bridge WP Composite).

- The sculpting phase begins with installation before and after curing; specifically, after curing the bridge is shaved, shaped, shaved and contoured to a tooth like looking replacement $(25 \mu \mathrm{m}$ and $50 \mu \mathrm{m}$ flamed shaped finishing diamonds, Lasco Diamonds).

- The polishing and finishing phase ensures the proper aesthetics and bite relationship and surface gloss (Berlew polishing wheels, Dedico "white flexies" rubber wheels) (Table 1).

\begin{tabular}{|c|c|c|c|c|c|}
\hline & $\begin{array}{l}\text { Removable flipper (Maryland } \\
\text { bridge) }\end{array}$ & Traditional bridge & Dental Implant & $\begin{array}{l}\text { Direct Adhesive-DAB } \\
\left.\text { Bridge }{ }^{\circledR}\right)\end{array}$ & (Carlson \\
\hline Cost & $\sim \$ 1,740.00$ & $\sim \$ 4,500.00$ & $\sim \$ 6,400.00$ & $\sim \$ 1,600.00$ & \\
\hline No. of visits & $2-5$ & $3-5$ & $12-20$ & 1 & \\
\hline Definitive result & No & Yes & Yes & Yes & \\
\hline Approx. lifespan & 6 months- 1 year & 3-5 years & ? & $3-5$ years & \\
\hline Tissue altered & Little & Yes & Yes & None & \\
\hline Completion time & 1-2 Weeks & About 1 month & 6 months-1 year & One Day & \\
\hline Time in chair & 1 hour & $2-3$ hours & $2-5$ hours & 1.5 hours & \\
\hline Expected pain & No & Moderate to High & Moderate to High & No & \\
\hline Needed meds & No & Yes & Yes & No & \\
\hline Easily modified & No & No & No & Yes & \\
\hline Shade modified & No & No & No & Yes-Anytime & \\
\hline Laboratory costs & Yes & Yes-High & Yes-Very High & None & \\
\hline Stress doctor & Very little & Very High & Very High & Little/Moderate & \\
\hline Galvanic current & (Clasps)-2-3uA & (Metal)-3-12uA & (Tit.)-30-200uA1 & None & \\
\hline Stress patient & Very Little & Moderate/High & Very High & Little/None & \\
\hline Death threat & None & Moderate & Moderate to High & None & \\
\hline
\end{tabular}

Table 1: Single tooth replacement comparison.

\section{Case Study}

An 82 years old man split tooth \#12 and came to us to correct his missing tooth issue. He had heard of our tooth replacement process and wanted it done rather than extensive tooth reduction for a traditional bridge or implant placement for a crown. His physical condition prohibited long sessions in the chair since he had spinal scoliosis. In view of his requirements we accomplished the following steps as demonstrated in the photos as follow in one hour. In (Figure 1) a missing left first premolar, tooth \#12 is seen. The prefabricated "Winged Pontic" is seen in (Figure 2) having been adjusted for the edentulous space. It shows occlusal relationships of the prefab "Winged Pontic," trimmed and adjusted into the space between the support teeth without attachment composite affixed (Figure 3).

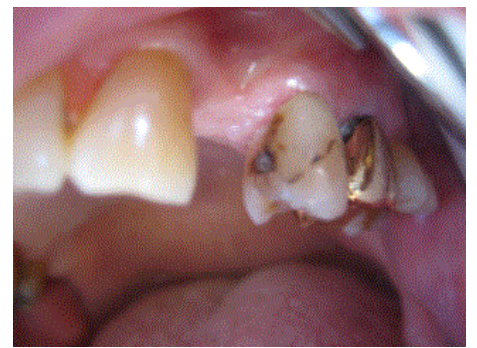

Figure 1: Pre-op showing slight decay on mesial of \#13 with a cervical composite restoration on the facial aspect. 
Page 3 of 4

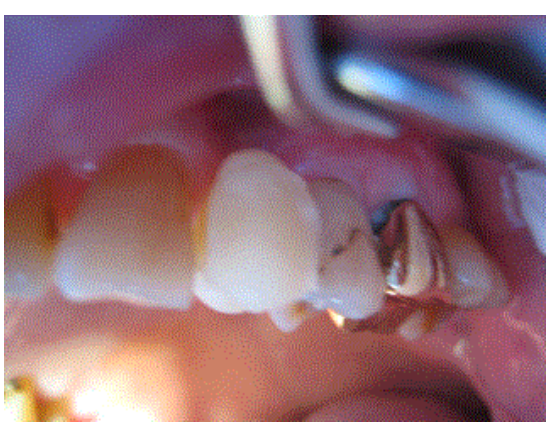

Figure 2: The "Winged Pontic" is shown after fitting into the edentulous area.

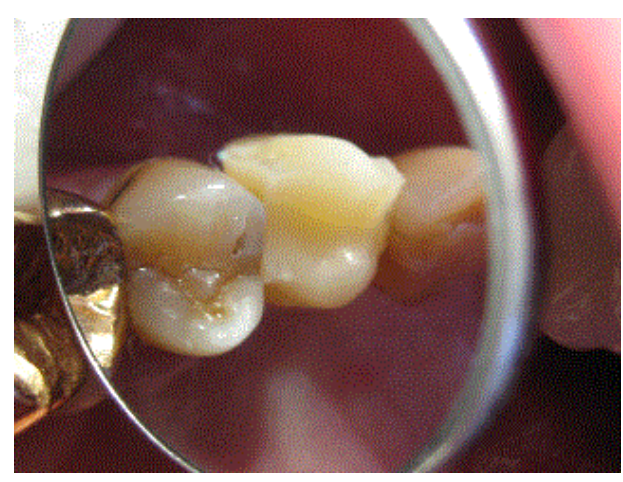

Figure 3: Occlusal view of loose fitting "Winged Pontic".

The line of placement is confirmed as the "Winged Pontic" is adjusted to flow in and out of the space easily.

Figure 4 shows the "Winged Pontic" first premolar \#12 with attachment composite applied to the proximal surfaces ready for insertion between \#11 and \#13, but only after preparation of the support teeth \#11 and \#13 for decay and surface cleanliness.

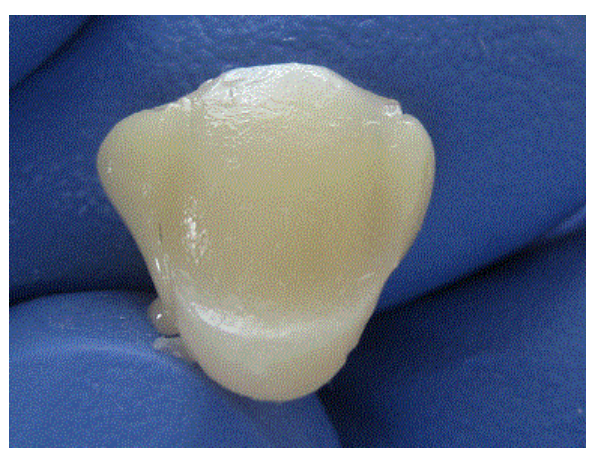

Figure 4: "Winged Pontic" prepared for installation with soft composite on Proximals.

After treating the enamel of support teeth \#11 and \#13 with etchant, water cleaning and drying, old existing composites are silanated with Carlson Bridge "Winged Pontic" Bond Enhancer and then a coat of clear resin and attachment composite are applied to proximal surfaces (Figure 5).

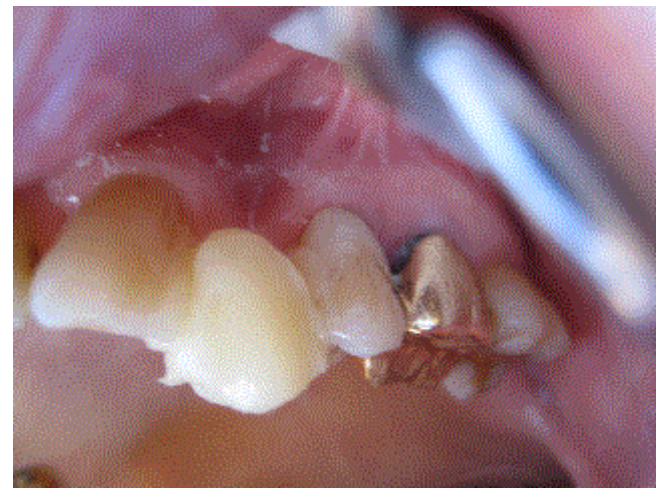

Figure 5: Once proximals of $\# 11$ and $\# 13$ are readied, the "WP" is installed.

The prefab "Winged Pontic" has first been etched for 30 seconds, cleaned with water, next treated with the $\mathrm{CB}^{\star}$ "WP" Bond Enhancer, clear resin is applied, followed by attachment composite application to proximal surfaces (Figure 4). The "Winged Pontic" is now ready for insertion between the support teeth (Figure 5). The prefabricated, pretreated "Winged Pontic" is carried to the space and inserted in the predetermined line of draw (Figure 5 and 6). The attachment composite is smoothed over all aspects of the pontic and support teeth and then light cured. The "Winged Pontic" is layered over with finishing composite, if needed. This is a time when creative artistic skills may be applied. Once cured, the occlusion is checked and adjusted in all excursions. Various flame shaped finishing diamonds are used for characterization (Figure 6).

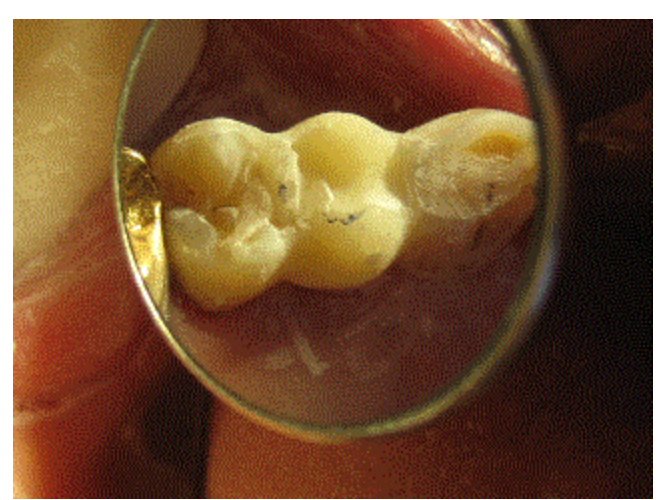

Figure 6: Once full insertion complete curing takes place and adjusting begins.

Final polish may be done with fine finishing diamonds 25 micron and 50 micron, and rubber wheels. Final results are demonstrated in (Figure 7 and 8). 


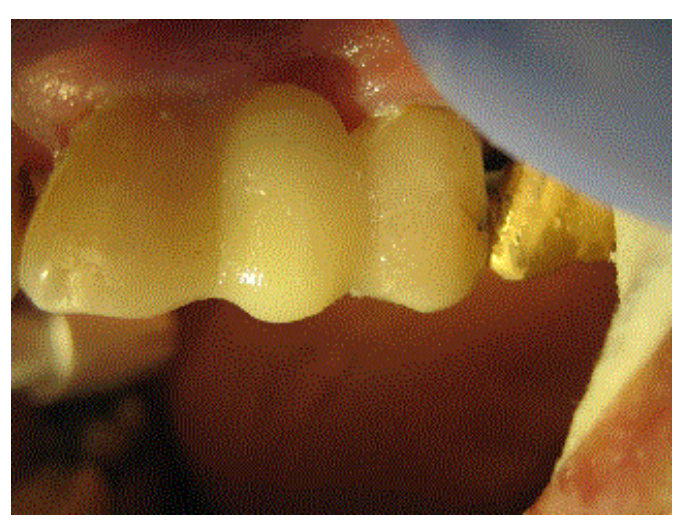

Figure 7: Final adjusting and polishing of the Carlson Bridge "WP".

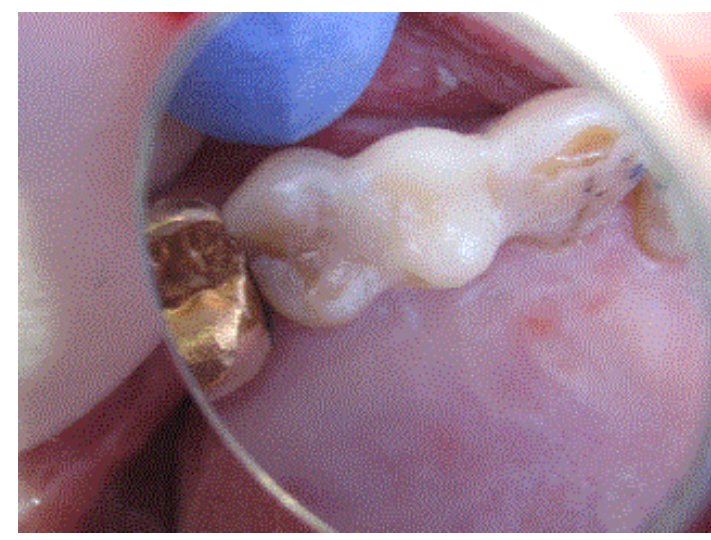

Figure 8: Occlusal view of the finsihed composite bridge.

\section{Summation}

This process, the Carlson Bridge "Winged Pontic" tooth replacement system may open doors to new possibilities in the way dentists practice [6]. Biosynthetic tissue engineering seems to be the leading edge in dentistry today, but only as an adjunct to other technical procedures carried out in the dental office, such as implants, flippers, or traditional porcelain veneer bridges. Resin composite materials are the closest to dentine and enamel in their strengths witnessed by their flexural modulus, compressive strengths and wear capacities. With this new methodology, it may also be a pleasant experience for the patient who can shorten his or her time in the dental chair and come away with an immediate dental cosmetic enhancement. Additionally, it will not create a financial burden or, require extensive healing time or unnecessary oral discomfort. It is also beneficial to the dental practitioner since the procedure is shorter in duration, therefore physically less demanding, less complicated in that intricate tooth preparations are unnecessary, and ultimately more rewarding creatively, artistically and remuneratively [7]. As with the new restorative composite systems of direct composite restorations the "Winged Pontic" prefab system offers a choice for the doctor and the patient regarding complex treatment plans and procedures. In this day and age of limited dental health budgets, it is an idea whose time has come. In senior populations health issues as well as costs are a major consideration in replacement of missing teeth. We have perfected the methodology for a one appointment, minimal time in the chair, direct composite bridge we identify as the Carlson Bridge "Winged Pontic" tooth replacement system for one or more missing teeth. We present this short article with the intention of encouraging others to use this non-invasive, artistically satisfying and relatively inexpensive system not only for seniors, special needs people, but in young and middle age people as well.

\section{References}

1. Jokstad A, Gokce M, Hjortsjo C (2005) A systematic review of the scientific documentation of fixed partial dentures made from fiberreinforced polymer to replace missing teeth. Int J Prosthodont 18: 489-496.

2. Knight JS, Whittaker DA (2003) A new look at chair-side fiber reinforcement of resin composite. Gen Dent 51: 334-336.

3. Carlson RS (1999) Breakthrough dental bridgework: The bio-logical dental bridge. Dentistry Today 18: 88-93.

4. van Heumen CC, Kreulen CM, Bronkhorst EM, Lesaffre E, Creugers NH (2008) Fiber-reinforced dental composites in beam testing. Dent Mater 24: $1435-1443$.

5. Carlson RS (2003) Dental artistry: General dentistry. J A Gen Dent 51: 4.

6. Carlson RS (2014) Preformed design bridging concept: A Case Report. Dent Today 33: 124-127.

7. Bayne SC (2005) Dental biomaterials: Where are we and where are we going? J Dent Educ 69: 571-585. 